\title{
Scaled, patient-specific 3D vertebral model reconstruction based on 2D lateral fluoroscopy
}

\author{
Guoyan Zheng · Lutz-P. Nolte · Stephen J. Ferguson
}

Received: 11 January 2010 / Accepted: 30 June 2010 / Published online: 20 July 2010

(C) CARS 2010

\begin{abstract}
Backgrounds Accurate three-dimensional (3D) models of lumbar vertebrae are required for image-based 3D kinematics analysis. MRI or CT datasets are frequently used to derive 3D models but have the disadvantages that they are expensive, time-consuming or involving ionizing radiation (e.g., CT acquisition). An alternative method using 2D lateral fluoroscopy was developed.

Materials and methods A technique was developed to reconstruct a scaled 3D lumbar vertebral model from a single twodimensional (2D) lateral fluoroscopic image and a statistical shape model of the lumbar vertebrae. Four cadaveric lumbar spine segments and two statistical shape models were used for testing. Reconstruction accuracy was determined by comparison of the surface models reconstructed from the single lateral fluoroscopic images to the ground truth data from 3D CT segmentation. For each case, two different surface-based registration techniques were used to recover the unknown scale factor, and the rigid transformation between the reconstructed surface model and the ground truth model before the differences between the two discrete surface models were computed.

Results Successful reconstruction of scaled surface models was achieved for all test lumbar vertebrae based on single lateral fluoroscopic images. The mean reconstruction error was between 0.7 and $1.6 \mathrm{~mm}$.

Conclusions A scaled, patient-specific surface model of the lumbar vertebra from a single lateral fluoroscopic image can be synthesized using the present approach. This new method for patient-specific 3D modeling has potential applications in spine kinematics analysis, surgical planning, and navigation.
\end{abstract}

G. Zheng $(\varangle) \cdot$ L.-P. Nolte $\cdot$ S. J. Ferguson

Institute for Surgical Technology and Biomechanics,

University of Bern, Stauffacherstrasse 78, Bern 3014, Switzerland

e-mail: guoyan.zheng@ieee.org; guoyan.zheng@istb.unibe.ch
Keywords Lumbar vertebra - Reconstruction · X-ray · 2D/3D - Kinematic analysis · Lateral fluoroscopic image

\section{Introduction}

Several studies have shown that fluoroscopy is well suited to in vivo lumbar spine kinematics analysis due to its capability of screening patients during free motion with an acceptably low radiation dosage [1,2]. The disadvantage of this technique, however, lies in its limitation to planar motion analysis. To enable fluoroscopic image-based 3D kinematic analysis, accurate three-dimensional (3D) models are needed $[3,4]$. If kinematics of an implanted prosthesis is the interest, a computer-aided design (CAD) model can be used [5]. However, this is not the case for analyzing in vivo lumbar spine kinematics. Thus, MRI or CT datasets are frequently used to derive 3D models but have the disadvantages that they are expensive, time-consuming or involve ionizing radiation (e.g., CT acquisition). In this paper, we present a technique to reconstruct a scaled 3D lumbar vertebral model from a single two-dimensional (2D) lateral fluoroscopic image.

Constructing a 3D surface model of the vertebra from $2 \mathrm{D}$ calibrated fluoroscopic image(s) is a challenging task. A priori information is often required to handle this otherwise ill-posed problem. Previously, kriging-based methods [6-11] as well as statistical shape model (SSM)-based methods [12-14] have been proposed.

Kriging-based methods start with identification of a predefined set of anatomical landmarks in one or more radiographs. The reconstruction is done by first estimating the scale and the rigid transformation of a generic object with respect 
to those identified landmarks and then applying a kriging algorithm to deform the generic object to obtain the final surface model. A limitation common to most kriging-based methods lies in the requirement of the interactive identification of landmarks. For example, Aubin et al. [6] required that a set of 6 stereo-corresponding points per vertebra should be identified, while in a later work from the same group [7], this set was increased even more to include landmarks that were visible in only one of the radiographs. Because such an interactive landmark identification process is time-consuming, error-prone, and user-dependent, statistical correlationbased methods have been proposed to reduce the number of landmarks that are required to be identified from the X-ray radiographs for decreasing the reconstruction time. Using a linear regression model, Pomero et al. [8] reduced the number of landmarks to a set of four landmarks per vertebra per radiograph such that the reconstruction time was decreased to less than 20 min. Both Humbert et al. [9] and Dumas et al. [10] tried to reduce user interaction by requesting the identification of the spine midline on two radiographs and by making use of statistical data for inferring the spine shape. An average identification time of $2.5-5 \mathrm{~min}$ was reported $[9,10]$. The more recent work published by Kadoury et al. [11] also started with the identification of the spine midline on two radiographs. The personalized $3 \mathrm{D}$ reconstruction of the spine was then achieved with the help of a segmentation algorithm, which took into consideration the variable appearance of scoliotic vertebrae from standard quality images in order to segment and isolate individual vertebrae on the radiographic planes.

Instead of using one generic object as the prior information, the methods in the second category use statistical shape models obtained from statistical shape analysis. Statistical shape analysis is an important tool for understanding anatomical structures from medical images [15-17]. Statistical shape models give efficient parameterization of the shape variations found in a collection of sample models of a given population. Model-based approaches are popular due to their ability to robustly represent objects $[18,19]$. In Benameur et al. [12,13], a SSM of scoliotic vertebrae was fitted to two radiographic views by simultaneously optimizing both shape and pose parameters. The optimal estimation was obtained by iteratively minimizing a combined energy function, which is the sum of a likelihood energy term measured from an edge potential field on the images and a prior energy term measured from the statistical shape model. Boisvert et al. [14] used a statistical articulated model of the spine for 3D reconstruction from partial radiographic data. Previously, we proposed a 2D-3D reconstruction scheme combining statistical instantiation and regularized shape deformation with an iterative image-to-model correspondence-establishing algorithm and showed its application to reconstruct a surface model of the proximal femur $[20,21]$.
Common to all these previous works is that at least two images are used as the input. Recently, we proposed a novel technique that could reconstruct a scaled, patient-specific 3D surface model from a standard X-ray radiograph and showed its application to reconstruct a surface model of the pelvis [22]. Based on this work, this paper presents an improved technique that combines a landmark-to-ray registration with a statistical shape model-based 2D/3D reconstruction scheme for reconstructing a scaled, patient-specific 3D surface model of the lumbar vertebra from a single fluoroscopic image. The landmark-to-ray registration is used to find an initial scale and an initial rigid transformation between the fluoroscopic image and the statistical shape model. The estimated scale and rigid transformation are then used to initialize the statistical shape model-based 2D/3D reconstruction scheme. The differences between the present work and the works we introduced previously [20-22] are as follows: (a) in our previous works [20-22], the image contours were manually extracted by interactively picking points from the X-ray images, while in the present work, we propose to use a semiautomatic segmentation tool based on the Livewire algorithm [23]. The crucial point of implementing the Livewire algorithm is the construction of its local cost function. The local cost function in this work is calculated from two components: multiscale gradient magnitudes and gradient direction and (b) our single X-ray image-based 2D/3D reconstruction technique has only been applied to derive a surface model of a single anatomy, i.e., the pelvis, while in the present work, we would like to adapt it to reconstruct a surface model of the lumbar vertebra using statistical shape models that are constructed from a set of training lumbar vertebral models across five lumbar levels.

This paper is organized as follows. The construction of the statistical shape models is discussed briefly in the following section. This is then followed by the sections that describe the statistically deformable $2 \mathrm{D} / 3 \mathrm{D}$ reconstruction approach and experimental design. Finally, the experimental results, discussion and conclusions are provided.

\section{Construction of the statistical shape models of the lumber vertebrae}

In this work, two statistical shape models were constructed from two databases consisting of different numbers of training lumbar vertebral models. Our goal was to construct statistical shape models of the lumbar vertebrae, simultaneously considering shape information from all five lumbar levels, and thereby to determine the principal modes of shape variation. We chose the point distribution model (PDM) [19] as the representation of the SSMs of the lumbar vertebrae. The reason why we constructed two statistical shape models was because there was significant shape difference between the 
lumbar vertebrae contained in the first three of the four test lumbar spine segments used in the present study and those in the fourth test lumbar spine segment (see Sect. "Experimental design" below for details). More specifically, all the lumbar vertebrae in the first three of the four test lumbar spine segments have broken transverse processes, while all the lumbar vertebrae in the fourth test lumbar spine segment have com- plete structures. Thus, the first PDM was constructed from a training database consisting of 11 CT-segmentation-based binary volumes of lumbar vertebrae with broken transverse processes (the broken-vertebra database) and was named as the broken-vertebra PDM (according to the spine level, the distribution of these 11 binary volumes are as follows, L1 level: 1; L2 level: 3; L3 level: 3; L4 level: 3, and L5 level: 1).
Fig. 1 The first two eigenmodes of variation of the two PDMs used in the present work. The shape instances from left to right at each row were generated by evaluating $\overline{\mathbf{x}}+\alpha \sigma_{k} \mathbf{p}_{k}$, with $\alpha \in\{-2,-1,1,2\}$. a Front view of the broken-vertebra PDM ( $k=1$ : the 1 st row; $k=2$ : the 2nd row), b lateral view of the broken-vertebra PDM $(k=1$ : the 1 st row; $k=2$ : the 2 nd row); c front view of the complete-vertebra PDM $(k=1$ : the 1 st row; $k=2$ : the 2 nd row); and $\mathbf{d}$ lateral view of the complete-vertebra PDM $(k=1$ : the 1st row; $k=2$ : the 2 nd row) (a)
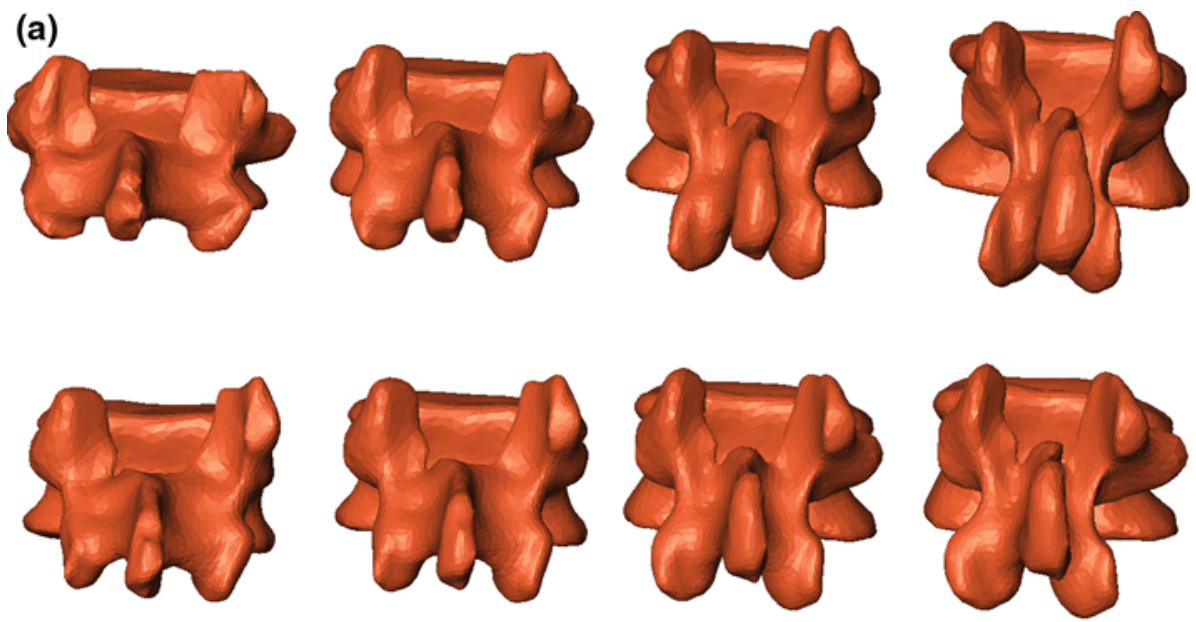

(b)
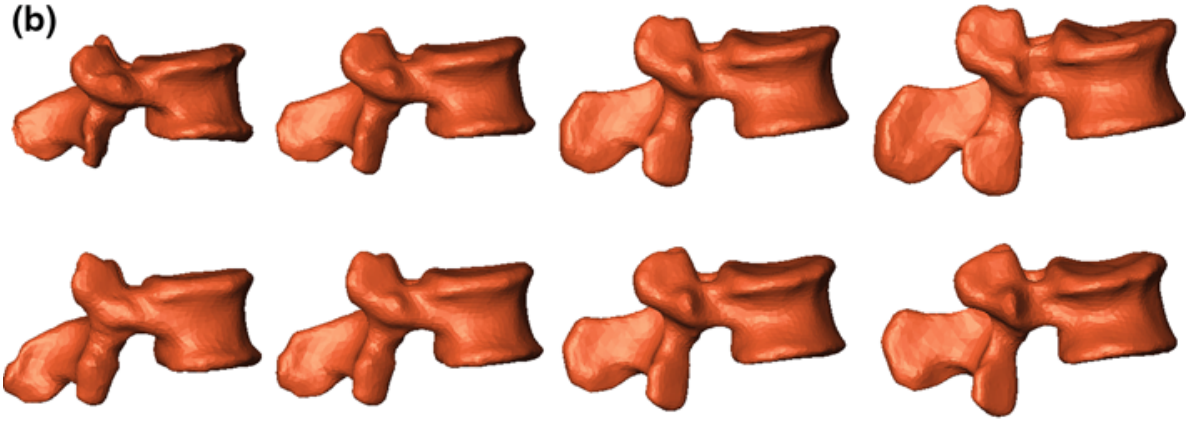

(c)
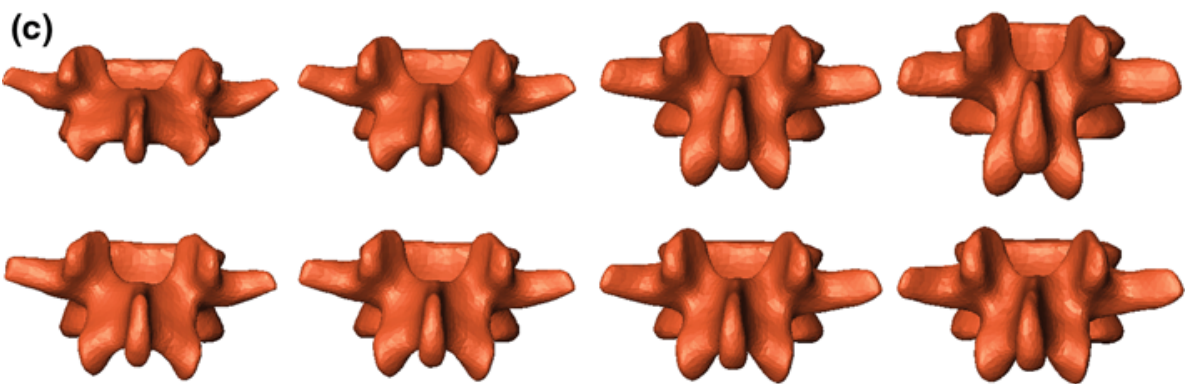

(d)
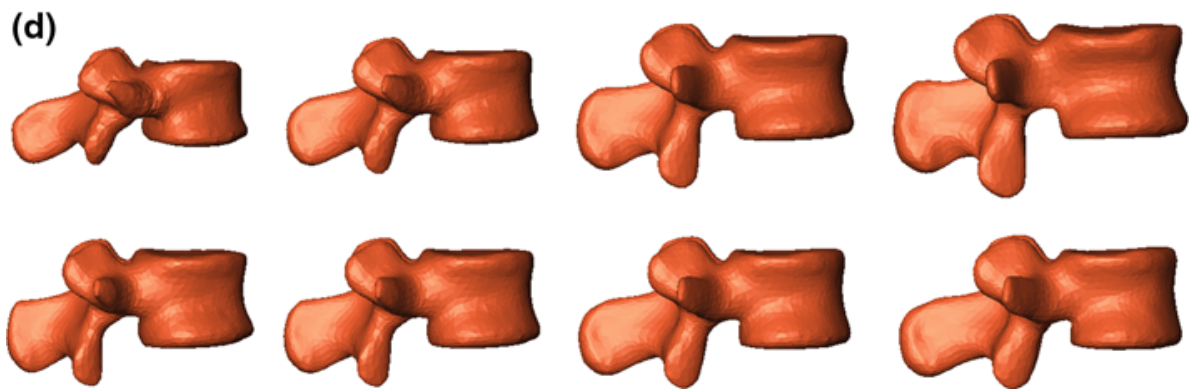
The second PDM was constructed from a training database consisting of 39 CT-segmentation-based binary volumes of lumbar vertebrae with complete structures (the completevertebra database) and was named as the complete-vertebra PDM (according to the spine level, the distribution of these 39 binary volumes are as follows, L1 level: 3; L2 level: 5; L3 level: 9; L4 level: 14; L5 level: 8). Although different training data were used, the exact same construction procedure was applied in the present work to construct the two PDMs. More specifically, a binary volume of a L3 level vertebra in the associated training database was chosen as the reference. Demon's algorithm, as implemented in MedINRIA [24], was used to estimate the deformation fields between the chosen reference binary volume and the other floating volumes in the associated database, i.e., for construction of the broken-vertebra PDM, we need to find the deformation fields between the reference binary volume chosen from the broken-vertebra database and the other 10 floating volumes, while for construction of the complete-vertebra PDM, we have to find the deformation fields between the reference binary volume chosen from the complete-vertebra database and the other 38 floating volumes. Each estimated deformation field was then used to displace the positions of the vertices on the reference surface model to the associated target volume. We thus obtained two sets of aligned surface models with established correspondences, with the first set consisting of 11 aligned surface models and the other set containing 39 aligned surface models.

Following the alignment, the two PDMs were constructed as follows. Let $x_{i}, i=0,1, \ldots, m-1$, be $m$ (here $m=11$ for the broken-vertebra PDM or $m=39$ for the completevertebra PDM) members of the aligned training surface models. Each member is described by a vector $\mathrm{x}_{i}$ with $N$ (here $N=5000$ for both the broken-vertebra PDM and the complete-vertebra PDM) vertices:

$\mathrm{x}_{i}=\left\{x_{0}, y_{0}, z_{0}, x_{1}, y_{1}, z_{1}, \ldots, x_{N-1}, y_{N-1}, z_{N-1}\right\}$

A PDM was then obtained by applying principal component analysis [25] to the associated training surface models:

$$
\begin{aligned}
& \mathbf{D}=\left((m-1)^{-1}\right) \cdot \sum_{i=0}^{m-1}\left(\mathrm{x}_{i}-\overline{\mathbf{x}}\right)\left(\mathrm{x}_{i}-\overline{\mathbf{x}}\right)^{T} \\
& P=\left(\mathbf{p}_{0}, \mathbf{p}_{1}, \ldots\right) ; \quad \mathbf{D} \cdot \mathbf{p}_{k}=\sigma_{k}^{2} \cdot \mathbf{p}_{k}
\end{aligned}
$$

where $\overline{\mathbf{x}}$ and $\mathbf{D}$ are the mean vector and the covariance matrix of the associated PDM, respectively. $\left\{\sigma_{k}^{2}\right\}$ is the non-zero eigenvalues of the covariance matrix $\mathbf{D}$, and $\left\{p_{k}\right\}$ is the corresponding eigenvectors. The descendingly sorted eigenvalues $\sigma_{k}^{2}$ and the corresponding eigenvector $p_{k}$ are the principal directions spanning a shape space with $\overline{\mathbf{x}}$ representing its origin. Figure 1 shows the variability captured by the first two modes of variations of the two PDMs.

\section{Statistically deformable 2D/3D reconstruction}

Without loss of generality, here we assume that the input image is calibrated and image distortion is corrected. For more details about fluoroscopic image calibration, we refer to our previous work [26]. Thus, for a pixel in the input image, we can always find a projection ray emitting from the focal point of the image through the pixel.

The single image-based surface model reconstruction technique proposed in this paper is based on a hybrid $2 \mathrm{D} / 3 \mathrm{D}$ deformable registration process, coupling a landmark-based scaled rigid registration with an adapted SSM-based 2D/3D reconstruction algorithm [20,21]. Different from the situation in our previous works [20,21], where two or more calibrated X-ray images were required as the input for a successful reconstruction, here only a single lateral fluoroscopic image is available. Similar to the situation when multiple images are used, the convergence of the single image-based 2D/3D reconstruction also depends on the initialization and on the image contour extraction. Thus, in the following, we focus on the image contour extraction and on a landmark-based scaled rigid registration for initializing the single image-based 2D/3D reconstruction.

\section{Image contour extraction}

As a feature-based 2D/3D reconstruction approach, our technique requires a pre-requisite image contour extraction. Explicit and accurate contour extraction is a challenging task, especially when the shapes involved become complex or when the background of the image becomes complex. In this paper, we feel that it is a far better choice to provide the user with a tool that supports interactive segmentation but at the same time speeds up the tedious manual segmentation process and makes the results repeatable. This leads us to developing a semiautomatic segmentation tool.

Our semiautomatic segmentation tool is based on the Livewire algorithm introduced by Mortensen and Barrett [23]. In their paper, graph edges are defined as the connection of two 8 -adjacent image pixels. A local cost function is assigned to the graph edges to weight their probability of being included in an optimal path. In this work, we use two static feature components to form this cost function. The first component $f_{G}$ is calculated from Canny edge detectors [27] at three different scales (the standard deviations of the Gaussian smoothing operator in these three scales are 1.0, 2.0, and 3.0 , respectively) as follows.

Let us denote the edges extracted by the Canny edge detector at three different scales as $E^{1}(\mathbf{q}), E^{2}(\mathbf{q})$, and $E^{3}(\mathbf{q})$, respectively. $\left\{E^{i}(\mathbf{q}) ; i=1,2,3\right\}$ is defined as follows: if pixel $\mathbf{q}$ is a detected edge pixel at the $i$ th scale, then $E^{i}(\mathbf{q})=1$; otherwise, it equals to zero. Let us further denote the gradient magnitudes at different scales as $G^{1}(\mathbf{q})$, 
$G^{2}(\mathbf{q})$, and $G^{3}(\mathbf{q})$, respectively. Then, we have

$$
\begin{aligned}
f_{G}(\mathbf{q})= & \left(1.0-\frac{G^{1}(\mathbf{q})}{\max \left(G^{1}(\mathbf{q})\right)} \cdot E^{1}(\mathbf{q})\right) \\
& +\left(1.0-\frac{G^{2}(\mathbf{q})}{\max \left(G^{2}(\mathbf{q})\right)} \cdot E^{2}(\mathbf{q})\right) \\
& +\left(1.0-\frac{G^{3}(\mathbf{q})}{\max \left(G^{3}(\mathbf{q})\right)} \cdot E^{3}(\mathbf{q})\right)
\end{aligned}
$$

According to Eq. (3), if $\mathbf{q}$ is not a detected edge pixel at the $i$ th scale, a constant cost of 1.0 will be added to the cost function. Otherwise, the cost depends on the gradient magnitude: the bigger the magnitude, the smaller the cost.

The second component, the gradient direction $f_{D}(\mathbf{p}, \mathbf{q})$, is calculated according to the form proposed in the original paper [23], which is used to add a smoothness term to the contour definition by assigning high costs to sharp changes.

Finally, these two static features are combined by weighted summation to form a single statistic local cost function as follows

$l(\mathbf{p}, \mathbf{q})=0.6 f_{G}(\mathbf{q})+0.4 f_{D}(\mathbf{p}, \mathbf{q})$

where the weights for these two terms are empirically determined.

Based on the Livewire algorithm, the semiautomatic contour extraction starts with a seed point, which is interactively placed by the user with a click of the left mouse button. During the extraction, the user can add more seed points by clicking the left mouse button. A click of the right mouse button will finish the definition of one contour. After that, clicking the left mouse button again starts the extraction of a new contour. Figure 2 shows an example of how the livewire segmentation technique is used to extract contours from the input image.

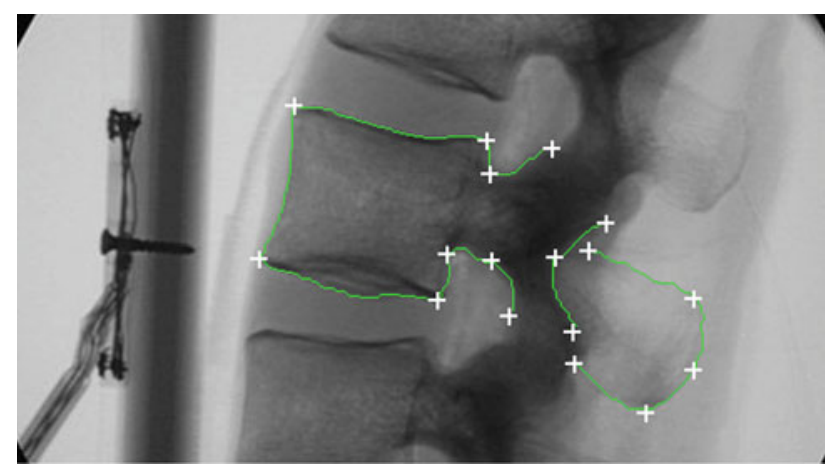

Fig. 2 Example of using livewire segmentation algorithm to extract image contours. The white crosses show where the user clicks the mouse button
Landmark-based scaled rigid registration for initialization

Initialization here means to estimate the initial scale and the rigid transformation between the mean model of the PDM and the input fluoroscopic image. For this purpose, we have adopted an iterative landmark-to-ray scaled rigid registration. The four anatomical landmarks that we used here are the center of the top surface of the vertebra body, the center of the bottom surface of the vertebra body, the geometrical center of the vertebra body, and the center of the spinal process tip. Their positions on the mean model of the PDM are obtained through point picking or center calculation (the center of the vertebra body is computed as the center of four boundary landmarks along the anterior-posterior direction as shown in Fig. 3a, while their positions on the fluoroscopic image are defined through interactive picking (see Fig. 3a and b for details).

Let us denote those landmarks defined on the mean model of the PDM, i.e., the vertebra body center, the center of the
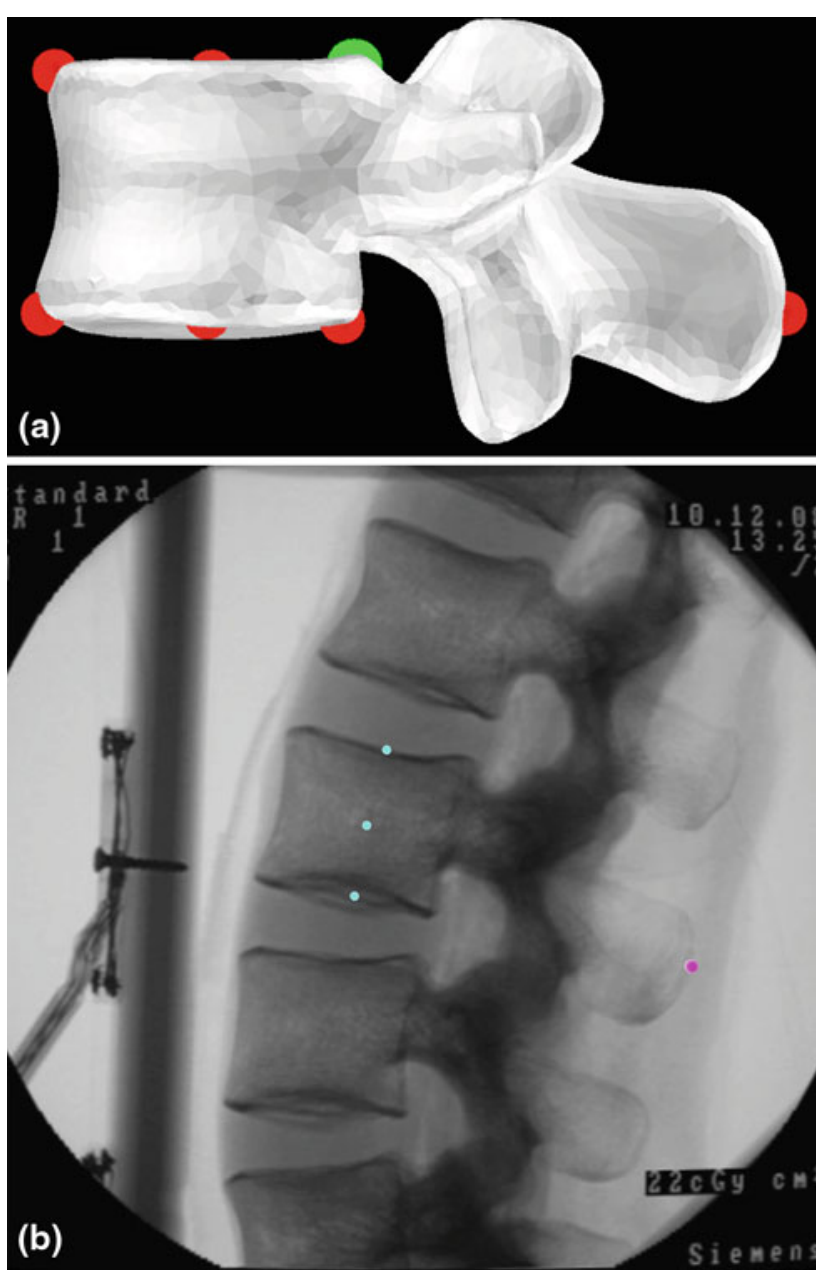

Fig. 3 Definition of initialization landmarks. a Landmarks extracted from the mean model of the PDM and $\mathbf{b}$ landmarks extracted from the fluoroscopic images 
top surface of the vertebra body, the center of the bottom surface of the vertebra body, and the center of the spinal process tip, as $v_{\text {Mean }}^{1}, v_{\text {Mean }}^{2}, v_{\text {Mean }}^{3}$, and $v_{\text {Mean }}^{4}$, respectively; and their corresponding landmarks interactively picked from the fluoroscopic image as $v_{\mathrm{X} \text {-ray }}^{1}, v_{\mathrm{X} \text {-ray }}^{2}, v_{\mathrm{X} \text {-ray }}^{3}$, and $v_{\mathrm{X} \text {-ray }}^{4}$, respectively. And for each X-ray landmark, we can calculate a projection ray emitting from the focal point to the landmark. We then calculate the length between $v_{\text {Mean }}^{1}$ and $v_{\text {Mean }}^{4}$ and denote it as $l_{\text {Mean }}^{1,4}$. Using the known image scale, we also calculate the length $l_{\mathrm{X} \text {-ray }}^{1,4}$ between $v_{\mathrm{X} \text {-ray }}^{1}$ and $v_{\mathrm{X} \text {-ray }}^{4}$. Then, we do:

\section{Data preparation}

In this step, we assume that the line connecting the centers of the vertebra body and the center of the spinal process tip is parallel to the input fluoroscopic image and is certain distance away from the imaging plane (in all the experiments reported in this paper, we used a fixed distance of $100 \mathrm{~mm}$ ). Using this assumption and the correspondences between the landmarks defined in the CT volume and those from the fluoroscopic image, we can compute two points $\bar{v}_{\mathrm{X} \text {-ray }}^{1}$ and $\bar{v}_{\mathrm{X} \text {-ray }}^{4}$ on the projection rays of $v_{\mathrm{X} \text {-ray }}^{1}$ and $v_{\mathrm{X} \text {-ray }}^{4}$, respectively (see Fig. 4a), which satisfy

$$
\begin{aligned}
& \bar{v}_{\mathrm{X} \text {-ray }}^{1} \bar{v}_{\mathrm{X} \text {-ray }}^{4} / / v_{\mathrm{X} \text {-ray }}^{1} v_{\mathrm{X} \text {-ray }}^{4} ; \quad \text { and } \\
& \left|\bar{v}_{\mathrm{X} \text {-ray }}^{1}-\bar{v}_{\mathrm{X} \text {-ray }}^{4}\right|=l_{\mathrm{X} \text {-ray }}^{1,4} \cdot \frac{F-d}{F}
\end{aligned}
$$

where "//" symbol indicates that the two lines are parallel; $F$ is the calibrated distance from the focal point to the imaging plane and $d$ is the assuming distance from the line connecting the center of the vertebra body and the center of the spinal process tip to the imaging plane.
The current scale $s$ between the mean model and the input image is then estimated as

$s=\left|\bar{v}_{\text {X-ray }}^{1}-\bar{v}_{\text {X-ray }}^{4}\right| / l_{\text {Mean }}^{1,4}$

Using $s$, we scale all landmark positions on the mean model and denote them as $\left\{\bar{v}_{\text {Mean }}^{i} ; i=1,2,3,4\right\}$. We then calculate the distances from $\bar{v}_{\text {Mean }}^{2}$ and $\bar{v}_{\text {Mean }}^{3}$ to the line $\bar{v}_{\text {Mean }}^{1} \bar{v}_{\text {Mean }}^{4}$ and denote it as $\bar{l}_{\text {Mean }}^{2,1-4}$ and $\bar{l}_{\text {Mean }}^{3,1-4}$, respectively.

Next, we find two points, point $\bar{v}_{\mathrm{X} \text {-ray }}^{2}$ on the projection ray of $v_{\mathrm{X} \text {-ray }}^{2}$ whose distance to the line $\bar{v}_{\mathrm{X} \text {-ray }}^{1} \bar{v}_{\mathrm{X} \text {-ray }}^{4}$ is equal to $\bar{l}_{\text {Mean }}^{2,1-4}$, and point $\bar{v}_{\mathrm{X} \text {-ray }}^{3}$ on the projection ray of $v_{\mathrm{X} \text {-ray }}^{3}$ whose distance to the line $\bar{v}_{\mathrm{X} \text {-ray }}^{1} \bar{v}_{\mathrm{X} \text {-ray }}^{4}$ is equal to $\bar{l}_{\text {Mean }}^{3,1-4}$. A paired-point matching based on $\left\{\bar{v}_{\text {Mean }}^{i} ; i=1,2,3,4\right\}$ and $\left\{\bar{v}_{\mathrm{X} \text {-ray }}^{i} ; i=1,2,3,4\right\}$ is used to calculate an updated scale $s_{0}$ and a rigid transformation $\bar{T}_{\text {Mean }}^{\mathrm{X} \text {-ray }}$ (see Fig. $4 \mathrm{a}$ for details). From now on, we assume that all information defined in the mean model coordinate frame has been transformed into the fluoroscopic image coordinate frame using $s_{0}$ and $\bar{T}_{\text {Mean }}^{\mathrm{X} \text {-ray }}$. We denote the transformed mean model landmarks as $\left\{\tilde{v}_{\text {Mean }}^{i} ; i=1,2,3,4\right\}$.

\section{Iteration}

The following steps are iteratively executed until convergence:

- $\quad$ For a point $\tilde{v}_{\text {Mean }}^{i}$, we find a point on the corresponding projection ray of $v_{\mathrm{X} \text {-ray }}^{i}$, which has the shortest distance to the point $\tilde{v}_{\text {Mean }}^{i}$ and denote it as $\tilde{v}_{\text {X-ray }}^{i}$ (see Fig. 4b). We then perform a paired-point matching using the extracted point pairs to compute a scale $\tilde{s}$ and a rigid transformation update $\Delta \tilde{T}_{\text {Mean }}^{\text {X-ray }}$.
Fig. 4 Iterative

landmark-to-ray registration.

a Schematic view of data preparation and $\mathbf{b}$ schematic view of finding $3 \mathrm{D}$ point pairs (a)

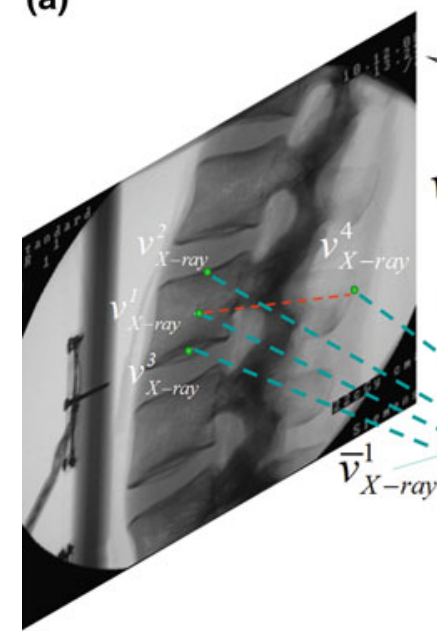

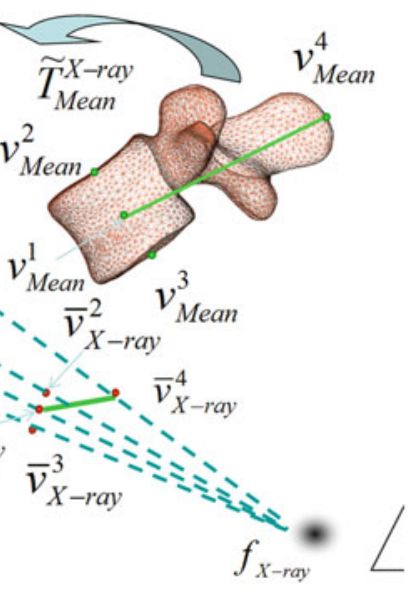

(b)

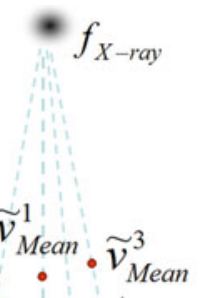

$\widetilde{v}_{\text {Mean }}^{2}$

$\widetilde{v}_{\text {Mean }}^{4}{ }^{\circ r_{0}} \widetilde{v}_{X-\text { ray }}^{4}$

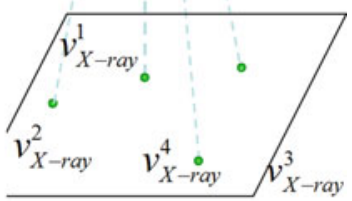


- We update the mean model coordinate frame using $\tilde{s}$ and $\Delta \tilde{T}_{\text {Mean }}^{\text {X-ray }}$.

In case when the landmark-based PDM initialization cannot guarantee the convergence of our 2D/3D reconstruction scheme that will be described below, we do provide an intuitive graphical user interface as described in our previous work [21] to allow fine-tuning of the scale and of the rotation of the mean model of the PDM with respect to the fluoroscopic image. The reasons why we only allow fine-tuning of the scale and of the rotation are because the scaling and the translating behavior along the projection direction are correlated to each other. For details about the graphic user interface, we refer to our previous work [21].

\section{Statistical shape model-based 2D/3D reconstruction}

The estimated scale and the rigid transformation between the mean model and the input image are then treated as the starting values for the PDM-based $2 \mathrm{D} / 3 \mathrm{D}$ reconstruction scheme [20,21], which depends on an iterative image-to-model correspondence-establishing algorithm that we introduced previously [28]. The image-to-model correspondence is established using a non-rigid $2 \mathrm{D}$ point matching process, which iteratively uses a symmetric injective nearest-neighbor mapping operator and 2D thin-plate splines-based deformation to find a fraction of best matched $2 \mathrm{D}$ point pairs between those contours extracted from the X-ray image as we described above and the projections of the apparent contours extracted from the 3D model. The apparent contours of a statistically instantiated 3D model are extracted using the approach introduced by Hertzmann and Zorin [29]. Previously, we mathematically proved that the proposed nonrigid 2D point matching process could automatically eliminate the cross-matching event [28], which was defined as the interactions between the lines linking any matched point pair. Figure 5a shows the mean mode of the complete-PDM initialized with respect to the input image using the landmark-based scaled rigid registration, and the apparent contours extracted from the mean model. An example of building 2D/2D correspondences between the image contours and the projections of the apparent contours of the mean model as shown in Fig. 5a is presented in Fig. 5b. The obtained 2D point pairs are then used to set up a set of 3D point pairs so that we turn a $2 \mathrm{D} / 3 \mathrm{D}$ reconstruction problem to a $3 \mathrm{D} / 3 \mathrm{D}$ one. For details about how the proposed non-rigid 2D point matching process works and about the mathematic proof of how the proposed process eliminates the cross-matching event, we refer to our previous work [28]. In the following, the details about how to convert the $2 \mathrm{D} / 3 \mathrm{D}$ reconstruction problem to a $3 \mathrm{D} / 3 \mathrm{D}$ one and how the latter problem is solved are given for completeness.
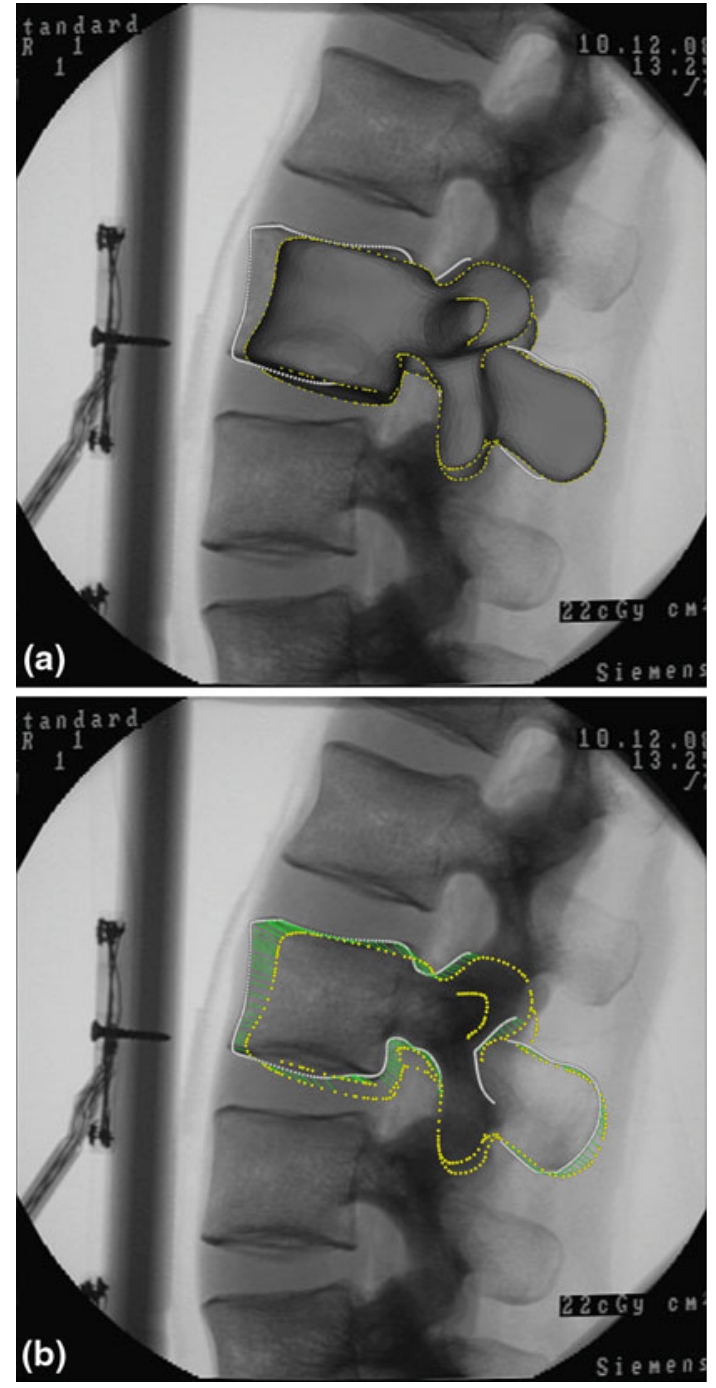

Fig. 5 Screenshots of establishing image-to-model correspondences. a The apparent contours (yellow dots) of the mean model (dark grey) of the complete-PDM after the landmark-based initialization and $\mathbf{b} 2 \mathrm{D} / 2 \mathrm{D}$ correspondences (green lines) between the image contours (white) and the projections of the apparent contours

\section{Converting a $2 D / 3 D$ problem to a $3 D / 3 D$ one}

Assume that a set of 2D matched point pairs $\left\{\left(A_{b}, I_{b}\right) ; b=\right.$ $0,1, \ldots, n-1\}$ have been found, where $A_{b}$ is the projection of a point on the apparent contours of a $3 \mathrm{D}$ model that is instantiated from the PDM and $I_{b}$ is a point on the image contours that is matched to $A_{b} ; n$ is the number of point pairs. The corresponding $3 \mathrm{D}$ point pairs are then constructed as follows (see Fig. 6 for a schematic illustration). For a 2D point $I_{b}$, one can find a projection ray $r_{b}$ emitting from the focal point of the X-ray image through the point $I_{b}$. Additionally, for its matched point $A_{b}$, one knows the associated 3D point $\Omega_{b}$ on the apparent contours of the model whose projection onto the image is $A_{b}$. By computing a point $v_{b}$ on the ray $r_{b}$ 


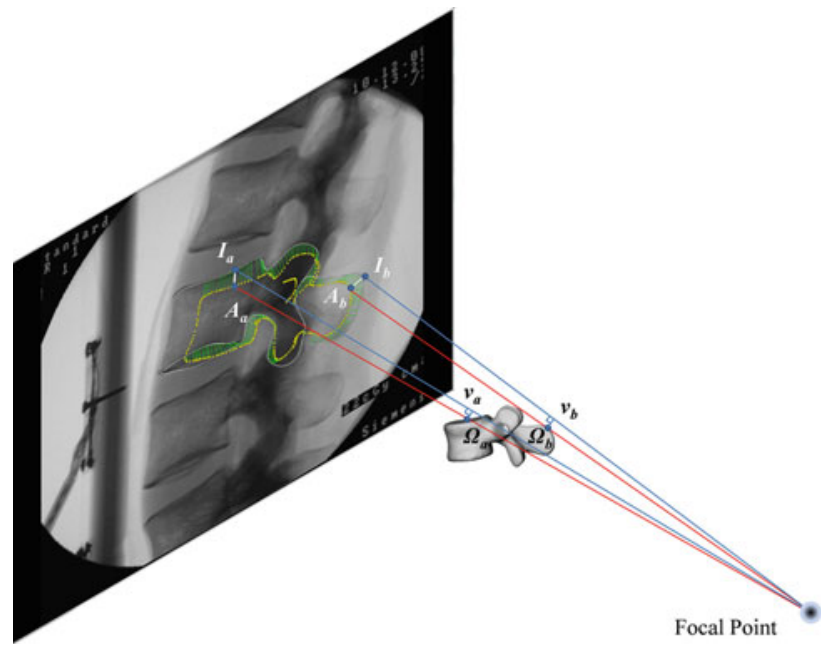

Fig. 6 Schematic illustration of computing 3D point pairs between a model and the input image from the established 2D/2D correspondences

that has the shortest distance to $\Omega_{b}$, a 3D point pair $\left(\Omega_{b}, v_{b}\right)$ can be obtained. Combining all these $3 \mathrm{D}$ point pairs, one can establish 2D/3D correspondence between the input image and a 3D model instantiated from the PDM, and thus convert a $2 \mathrm{D} / 3 \mathrm{D}$ reconstruction problem to a $3 \mathrm{D} / 3 \mathrm{D}$ one.

\section{$3 D / 3 D$ reconstruction}

As soon as a set of 3D point pairs are available, the problem of surface reconstruction is then solved optimally in three sequential stages using the algorithm presented in [28]: scaled rigid registration, statistical instantiation, and regularized shape deformation.

Scaled rigid registration This is the only stage that is solved iteratively. In this stage, the scale and the rigid registration transformation between the mean model of the PDM and the input images are iteratively determined using an adapted iterative closest point (ICP) algorithm [30]. The difference between this algorithm and the traditional ICP algorithm is that at each iteration, one need to set up a set of new point pairs using the algorithm for building $2 \mathrm{D} / 3 \mathrm{D}$ correspondences [28].

Statistical instantiation Based on the estimated scale and the pose information from the first stage, one can use the same correspondence-establishing algorithm to obtain a set of $n$ 3D point pairs from the input image. Let us denote the points computed from the image data as $\mathbf{v}^{\prime}=$ $\left\{v_{i}^{\prime}=\left(x_{i}^{\prime}, y_{i}^{\prime}, z_{i}^{\prime}\right) ; i=0,1, \ldots, n-1\right\}$ and call them the image points. Let us further denote those points on the mean model of the PDM as $\overline{\mathbf{x}}^{\prime}=\left\{\left(\overline{\mathbf{x}}_{j}\right)_{i} ; 0 \leq j \leq N-1\right.$; $i=0,1, \ldots, n-1\}$ and call them the model points, where $N$ is the number of points on the mean model; $\left(\overline{\mathbf{x}}_{j}\right)_{i}$ means that the $j$ th model point $\overline{\mathbf{x}}_{j}$ on the mean model $\overline{\mathbf{x}}$ is the closest point to the $i$ th image point $v_{i}^{\prime}$. The statistical instantiation is formulated as the minimization of the following joint cost function [28]:

$$
\left\{\begin{array}{l}
E_{\alpha}\left(\overline{\mathbf{x}}^{\prime}, \mathbf{v}^{\prime}, \mathbf{x}\right)=(\rho+\log (3 n)) \cdot E\left(\overline{\mathbf{x}}^{\prime}, \mathbf{v}^{\prime}, \mathbf{x}\right)+E(\mathbf{x}) \\
\mathbf{x}=\overline{\mathbf{x}}+\sum_{k=0}^{m-2} \alpha_{k} \cdot \sigma_{k} \cdot \mathbf{p}_{k} \\
E\left(\overline{\mathbf{x}}^{\prime}, \mathbf{v}^{\prime}, \mathbf{x}\right)=(1 / n) \\
\quad \sum_{i=0}^{n-1}\left\|v_{i}^{\prime}-\left(\left(\overline{\mathbf{x}}_{\mathrm{j}}\right)_{i}+\sum_{k=0}^{m-2} \alpha_{k} \cdot \sigma_{k} \cdot \mathbf{p}_{k}(j)\right)\right\|^{2} \\
E(\mathbf{x})=(1 / 2) \cdot \sum_{k=0}^{m-2}\left(\alpha_{k}^{2}\right)
\end{array}\right.
$$

where the first term $E\left(\overline{\mathbf{x}}^{\prime}, \mathbf{v}^{\prime}, \mathbf{x}\right)$ is the likelihood energy term and the second term $E(\mathbf{x})$ is the prior energy term, used to constrain the estimated shape to a realistic result. $\rho$ is a parameter that controls the relative weighting between these two terms. $\mathbf{p}_{k}(j)$ is the $j$ th tuple (a 3D vector) of the $k$ th shape basis eigenvector.

To determine $\left\{\alpha_{k}\right\}$, the cost function is differentiated with respect to the shape parameters and equated to zero, resulting in a linear system of $(m-1)$ unknowns, which can then be solved with standard methods. To generate the surface model from the calculated shape parameters, we adopted a strategy that was introduced in [28], i.e., a cutoff point was set such that only the first few modes before the cutoff point and their corresponding shape parameters were used in the model instantiation.

Regularized shape deformation In this stage, one needs to first set up the correspondences between the input images and a statistically instantiated surface model that is obtained through solving Eq. (5). To keep the same notation, let us assume that the image points in this stage are $\mathbf{v}^{\prime}=$ $\left\{v_{i}^{\prime}=\left(x_{i}^{\prime}, y_{i}^{\prime}, z_{i}^{\prime}\right) ; i=0,1, \ldots, l-1\right\}$ and that the model points in this stage are $\mathbf{v}=\left\{v_{i}=\left(\mathbf{x}_{j}\right)_{i}=\left(x_{i}, y_{i}, z_{i}\right)\right.$; $i=0,1, \ldots, l-1\}$, where $l$ is the number of the matched point pairs and $\left(\mathbf{x}_{j}\right)_{i}$ means that the $j$ th model point $\mathbf{x}_{j}$ on the statistically instantiated model $\mathbf{x}$ is the closest point to the $i$ th image point $v_{i}^{\prime}$. The regularized shape deformation is described as a regression problem of finding a 3D spatial transform $\mathbf{t}: \mathfrak{R}^{3} \rightarrow \mathfrak{R}^{3}$ that minimizes following cost function [28],

$$
E(\mathbf{t})=(1 / l) \cdot \sum_{i=0}^{l-1}\left\|v_{i}^{\prime}-\mathbf{t}\left(v_{i}\right)\right\|^{2}+\tau \cdot \frac{\log (m)}{\log (3 l)} \cdot L(\mathbf{t})
$$

where $\tau \geq 0$ is a parameter controlling the fitting quality and the regularization constraint; $m$ is the number of training surface models; $\mathbf{t}(\mathbf{v})=\left\{\mathbf{t}\left(v_{i}\right)\right\}$ are the results of applying the mapping on the model points and $L(\mathbf{t})$ is a thin-plate splines (TPS) kernel-based regularization functional defined on the 

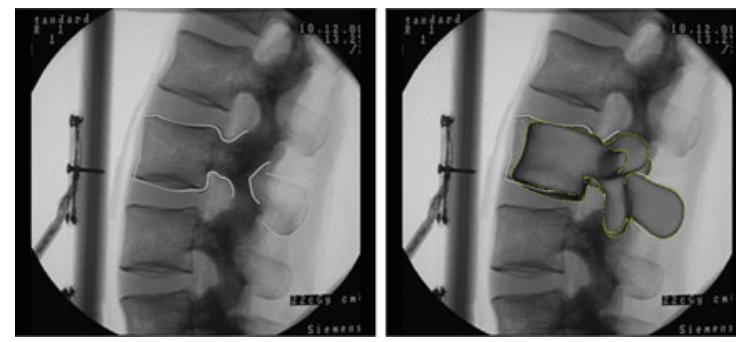

Fig. 7 Different stages of the reconstruction process. Left the smoothed contours; left middle the landmark-based initialization of the mean model (grey) of the PDM; right middle the reconstructed model

nonlinear mapping $\mathbf{t}$ and has following form [28],

$$
\left\{\begin{aligned}
L(\mathbf{t})= & \iiint_{\mathfrak{R}^{3}}(B(\mathbf{t})) \mathrm{d} x \mathrm{~d} y \mathrm{~d} z ; \quad \text { and } \\
B(\cdot)= & \left(\frac{\partial^{2}}{\partial x^{2}}\right)^{2}+2\left(\frac{\partial^{2}}{\partial x \partial y}\right)^{2}+\left(\frac{\partial^{2}}{\partial y^{2}}\right)^{2}+2\left(\frac{\partial^{2}}{\partial y \partial z}\right)^{2} \\
& +\left(\frac{\partial^{2}}{\partial z^{2}}\right)^{2}+2\left(\frac{\partial^{2}}{\partial z \partial x}\right)^{2}
\end{aligned}\right.
$$

For details about how to solve Eq. (6), I refer to our previous work [28]. Figure 7 shows different stages of the reconstruction process, where a scaled surface model of the L2 lumbar vertebra was reconstructed from a single lateral fluoroscopic image.

\section{Experimental design}

Four single lateral fluoroscopic images of four cadaveric lumbar spine segments (one image per cadaveric lumbar spine segment) were used to validate the present technique. In total, there were 15 lumbar vertebrae contained in these four spine segments, but only twelve of them were visible in the four lateral fluoroscopic images (three lumbar vertebrae per segment per image). Due to the reason that the transverse processes of all lumbar vertebrae (in total 11 lumbar vertebrae across different lumbar levels) contained in three of the four test lumbar spine segments were broken, CT-segmentation-based binary volumes of these 11 lumbar vertebrae with broken transverse processes were used to construct the broken-vertebra PDM as described in Sect. "Construction of the statistical shape models of the lumber vertebrae". The four lumbar vertebrae contained in the last spine segment had complete structures and their CT-segmentation-based binary volumes were used together with another 35 CT-segmentation-based binary volumes of lumbar vertebrae to construct the complete-vertebra PDM as presented in Sect. "Construction of the statistical shape models of the lumber vertebrae". All the binary volumes of the lumbar vertebrae contained in the test spine segments were semiautomatically segmented from the associated CT datasets using the commercially available software

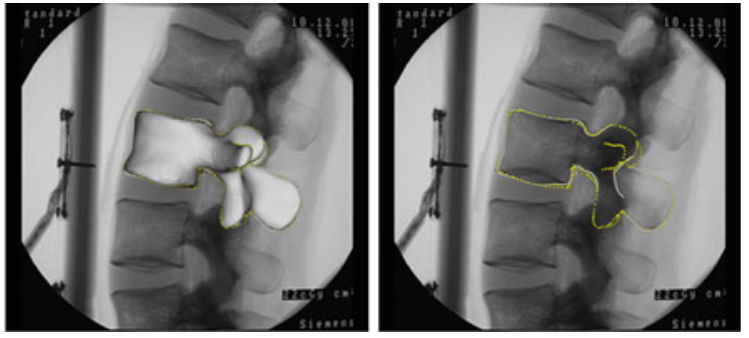

(white); right the apparent contours (yellow) extracted from the reconstructed model versus the image contours (white)

package Amira 5.0 (TGS Europe, Paris, France). To evaluate the reconstruction accuracy, a surface model derived from the binary volume of each test lumbar vertebra was used as the ground truths. As we only reconstructed a scaled surface model of the lumbar vertebra from each lateral fluoroscopic image, we had to first recover the unknown scale factor of the reconstructed model with respect to the ground truth surface model (or vice versa) before we could evaluate the reconstruction accuracy. For this purpose, we proposed to estimate the unknown scale factor of the reconstructed models by performing surface-based registrations [30]. After the registration, the open source tool MESH [31] was used to compute the distances between the reconstructed surface models and their associated ground truth surface models, which were regarded as the reconstruction errors. We adapted this tool to include the computation of different error statistics. To this end, based on the two PDMs and datasets of the four cadaver spine segments, we designed and conducted the following experiments:

- Experiment on evaluating the correspondence-establishing quality of the two PDMs.

- Experiment on evaluating the accuracy of the present technique in reconstructing the surface models of 9 lumbar vertebrae with broken transverse processes.

- Experiment on evaluating the accuracy of the present technique in reconstructing the surface models of 3 lumbar vertebrae with complete structures.

For all experiments, we used an Intel Duo Core 2.4- $\mathrm{GHz}$ laptop with $4 \mathrm{~GB}$ of RAM. All programming was done using Visual C++ 2005 on Windows Vista.

Experiment on evaluating the correspondence-establishing quality of the two PDMs

In statistical shape analysis, it is important to establish the correct correspondences, otherwise an inefficient parameterization of shape will be determined. It was reported in 
literatures $[32,33]$ that the goodness of the correspondence could be evaluated directly on the resultant SSM by following three measures:

- Model compactness It measures how compact a SSM is. According to Styner et al. [33], the compactness ability of a SSM is determined as the cumulative variance $C(M)=\sum_{i=1}^{M} \lambda_{i}$, where $\lambda_{i}=\sigma_{i}^{2}$ is the $i$ th eigenvalue. $C(M)$ is measured as a function of the number of shape parameters $M$. Furthermore, the standard error of $C(M)$ is determined from the training size $m: \sigma_{C(M)}=$ $\sum_{i=1}^{M} \sqrt{2 / m} \lambda_{i}$.

- Model generalization It measures the ability of a SSM to describe instances outside of the training set. More specifically, the generalization ability of a SSM is measured using leave-one-out reconstruction. A SSM is built using all but one member of the training set and then fitted to the leaved-out example. The accuracy to which the model can reconstruct the leave-out example is measured. The generalization ability is measured as a function $G(M)$ of the number of shape parameters $M$.

- Model specificity It measures the ability of a SSM to represent valid instances of the object class. According to Styner et al. [33], it is defined as the average distance of uniformly distributed, randomly generated objects in the model shape space to their nearest member in the training set. In this work, the number of random samples was chosen to be 1,000 .

Thus, the purpose of this experiment is to evaluate the correspondence-establishing quality of the two PDMs based on the above three measures.

Experiment on evaluating the accuracy of the present technique in reconstructing the surface models of 9 lumbar vertebrae with broken transverse processes

The purpose of this experiment is to evaluate the robustness and the accuracy of the present technique in reconstructing the surface models of 9 lumbar vertebrae ( $1 \mathrm{~L} 1$ level vertebra, 3 L2 level vertebra, 3 L3 level vertebra, and 2 L4 level vertebra) with broken transverse processes. To evaluate the accuracy, two different surface-based matching techniques, i.e., a surface-based anisotropically scaled rigid registration and a surface-based isotropically scaled rigid registration, were used to recover the unknown scale factor of the reconstructed surface mode with respect to its associated ground truth that was derived from a CT-reconstruction technique. Due to the reason that the broken-vertebra PDM was constructed from the CT-segmentation-based binary volumes of the test vertebrae, we designed two studies to evaluate the robustness and the accuracy of the present technique. The first study was called the leave-all-in study where all 11 aligned training surface models were used to construct the broken-vertebra PDM that was then used as the prior information to reconstruct the surface model of each test lumbar vertebra. Different from the first study, the second study was a leave-one-out study where the aligned training surface model corresponding to the current test vertebra was removed from the training database. A broken-vertebra PDM constructed from the leave-one-out training database was then used as the prior information to reconstruct the surface model of the left-out test vertebra.

Experiment on evaluating the accuracy of the present technique in reconstructing the surface models of 3 lumbar vertebrae with complete structures

This experiment was designed to evaluate the robustness and the accuracy of the present technique in reconstructing the surface models of lumbar vertebrae with complete structures. Unlike the situation in the last experiment, where all 9 test lumbar vertebrae had broken transverse processes, here all 3 test lumbar vertebrae (1 L1 level vertebra, $1 \mathrm{~L} 2$ level vertebra, and $1 \mathrm{~L} 3$ level vertebra) had complete structures. Similar to the situation in the last experiment, we also used the two different surface-based registration techniques to estimate the unknown scale factors between the reconstructed surface models and the associated ground truth surface models. Two studies were conducted in this experiment to evaluate the robustness and the accuracy of the present technique. Due to the reason that all 4 aligned training surface models of the lumbar vertebrae contained in the test spine segment were part of the training database for constructing the completevertebra PDM, we named the first study as the leave-all-in study. In this study, each time the complete-vertebra PDM as described in Sect. "Construction of the statistical shape models of the lumber vertebrae" was used together with the single lateral fluoroscopic image of the test spine segment to reconstruct a scaled surface model of a test vertebra. In the second study, all 4 aligned training surface models corresponding to the lumbar vertebrae in the test spine segment were removed from the training database, and a completevertebra PDM constructed from the rest 35 training surface models was used to reconstruct a scaled surface model of each test vertebra. We thus called the second study as the leave-four-out study.

\section{Results}

Results of the experiment on evaluating the correspondence-establishing quality of the two PDMs

Using the three measures, we evaluated the correspondenceestablishing quality of both PDMs. The results are presented 
in Fig. 8. Our compactness study showed that about $97 \%$ total amount of variations of the complete-vertebra PDM were explained by the first 20 eigenmodes and that it required the first 7 eigenmodes to describe the same amount of variations of the broken-vertebra PDM. Based on these results, in the following two experiments, we chose the cutoff point for the complete-vertebra PDM as 20 and the cutoff point for the
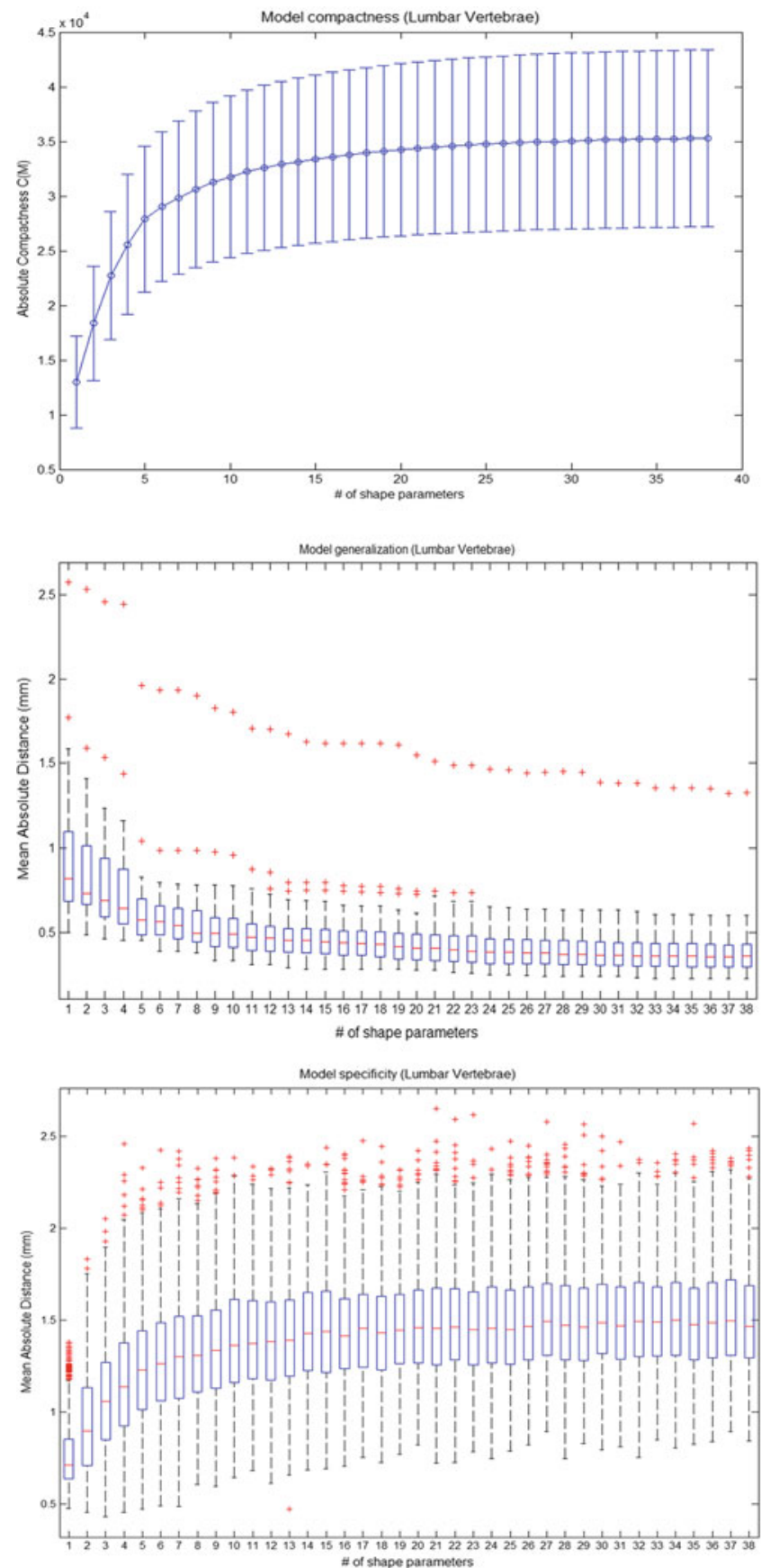

Fig. 8 Evaluating the correspondence-establishing quality of the two PDMs using three measures. Left column the complete-vertebra PDM; and right column the broken-vertebra PDM. At each column, top row broken-vertebra PDM as 7. The generalization study showed that both PDMs had the capability to represent unseen instance of the associated object class, while the specificity study demonstrated that both PDMs could generate instances of the associated object class in a reasonably good accuracy (i.e., all mean absolute distances in both specificity study are smaller than $1.5 \mathrm{~mm}$ ).
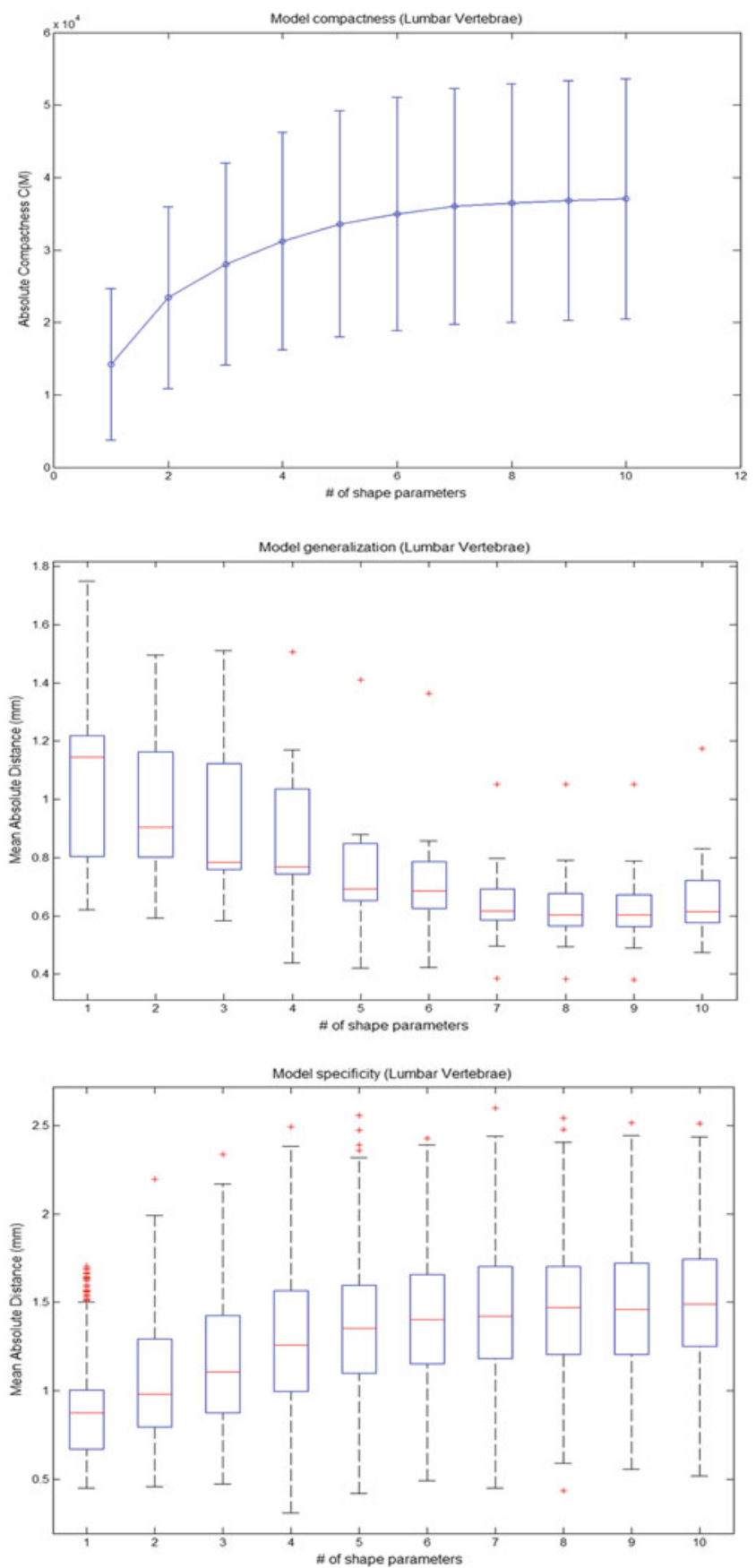

the compactness measures; middle row the generalization measures; and bottom row the specificity measures 
Results of the experiment on evaluating the accuracy of the present technique in reconstructing the surface models of 9 lumbar vertebrae with broken transverse processes

In both studies, the present technique could successfully reconstruct 3D surface models of all 9 test lumbar vertebrae with broken transverse processes. On average, it took the present technique about $90 \mathrm{~s}$ to finish the computation. The average errors of reconstructing surface models of all 9 vertebrae with broken transverse processes in both studies are shown in Table 1. A more detailed box-plot description of the reconstruction errors in both studies is shown in Fig. 9.
When the surface-based anisotropically scaled rigid registration was used to recover the unknown scale factors of the reconstructed surface models, an average mean reconstruction error of $0.9 \mathrm{~mm}$ (range: from 0.7 to $1.2 \mathrm{~mm}$ ) was found for the leave-all-in study and an average mean reconstruction error of $1.0 \mathrm{~mm}$ (range: from 0.8 to $1.3 \mathrm{~mm}$ ) was found for the leave-one-out study. In contrast, when the surfacebased isotropically scaled rigid registration was used, the average mean reconstruction error of the leave-all-in study was changed to $1.1 \mathrm{~mm}$ (range: $0.9-1.5 \mathrm{~mm}$ ) and the average mean reconstruction error of the leave-one-out study was changed to $1.2 \mathrm{~mm}$ (range: $0.9-1.6 \mathrm{~mm}$ ).

Table 1 Errors of reconstructing the surface models of the 9 lumbar vertebre with broken transverse processes

Vertebra Cadaver_2_L2 Cadaver_2_L3 Cadaver_2_L4 Cadaver_3_L1 Cadaver_3_L2 Cadaver_3_L3 Cadaver_4_L2 Cadaver_4_L3 Cadaver_4_L4

Leave-all-in study, when the anisotropically scaled rigid registration was used to recover the scale

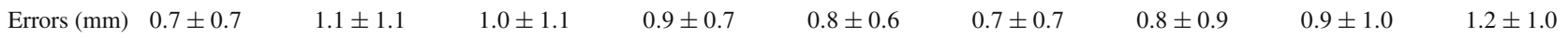

Leave-one-out study, when the anisotropically scaled rigid registration was used to recover the scale

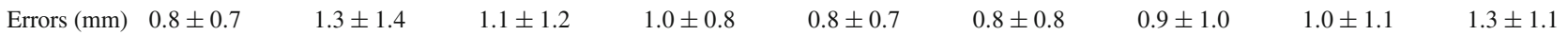

Leave-all-in study, when the isotropically scaled rigid registration was used to recover the scale

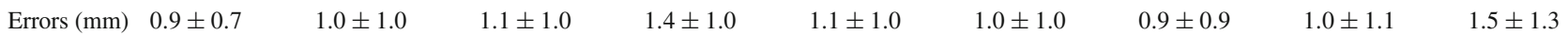

Leave-one-out study, when the isotropically scaled rigid registration was used to recover the scale

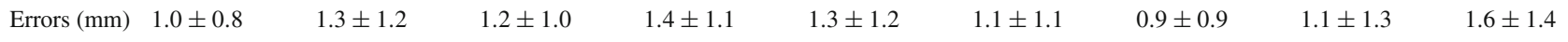
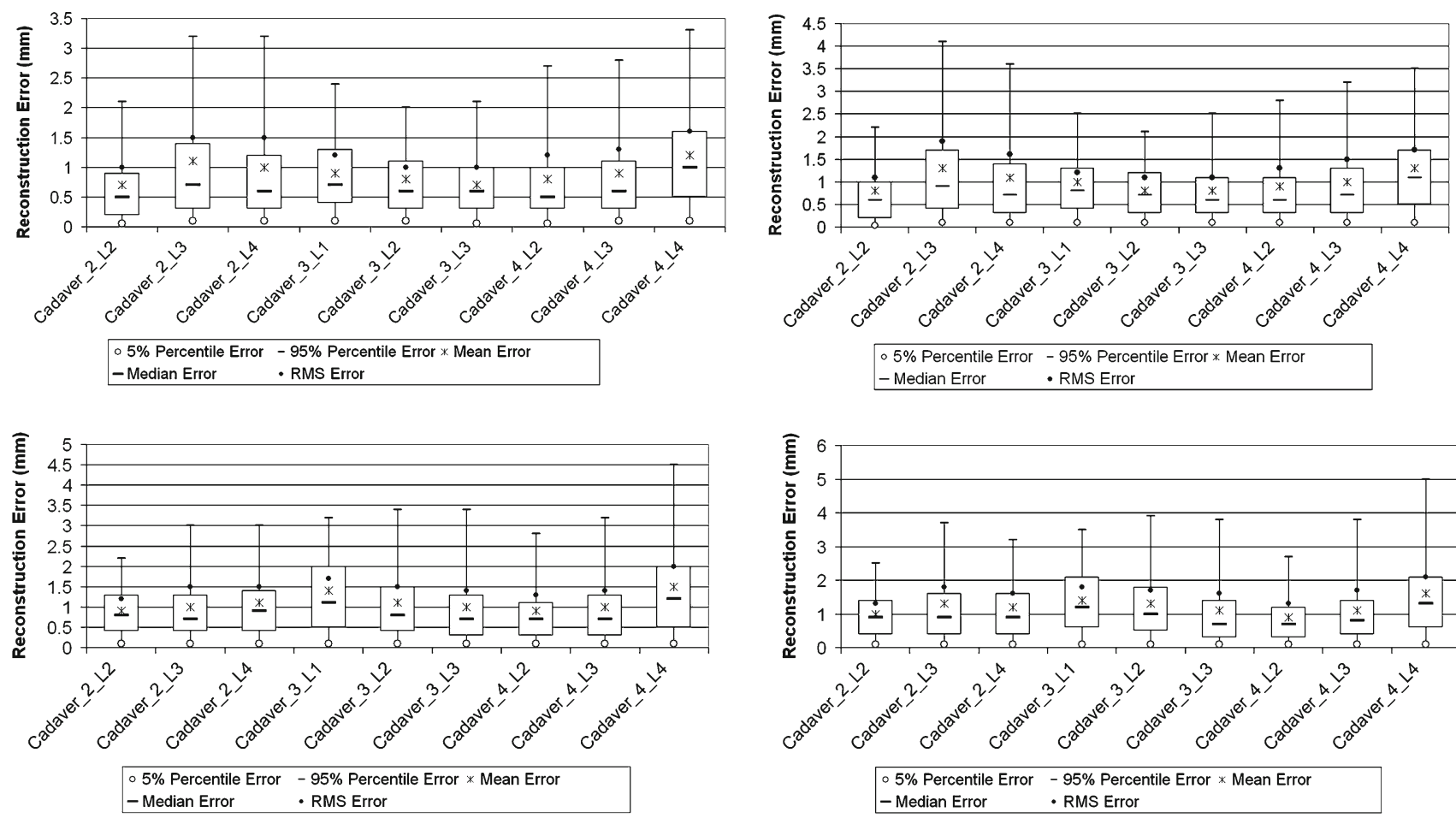

Fig. 9 Errors of reconstructing surface models of all 9 lumbar vertebrae with broken transverse processes when different surface-based matching techniques were used to recover the unknown scale factors between the reconstruct surface models and the associated ground truths. Top row when a surface-based anisotropically scaled rigid

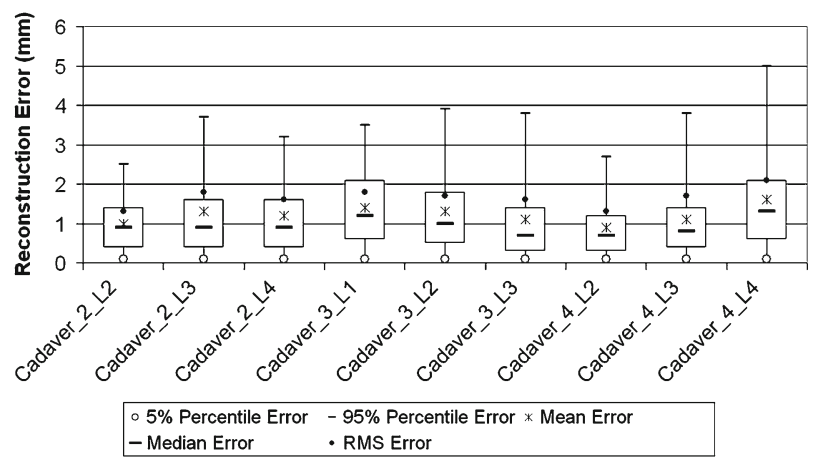

registration was used and bottom row when a surface-based isotropically scaled rigid registration was used. In both rows, the left column shows results of the leave-all-in study, while the right column shows the results of the leave-one-out study 
Results of the experiment on evaluating the accuracy of the present technique in reconstructing the surface models of 3 lumbar vertebrae with complete structures

In both studies, the present technique could successfully reconstruct 3D surface models of all 3 test lumbar vertebrae with complete structures. On average, it took the present technique about $105 \mathrm{~s}$ to finish the computation. The errors of reconstructing surface models of all 3 lumbar vertebrae with complete structures in both studies are shown in Table 2. A more detailed box-plot description of the reconstruction errors in both studies is shown in Fig. 10. When the surface-based anisotropically scaled rigid registration was used to recover the unknown scale factors, an average mean reconstruction error of $0.77 \mathrm{~mm}$ (range: from 0.7 to
$0.9 \mathrm{~mm}$ ) was found for the leave-all-in study and an average mean reconstruction error of $0.83 \mathrm{~mm}$ (range: from 0.8 to $0.9 \mathrm{~mm}$ ) was found for the leave-four-out study. In contrast, when the surface-based isotropically scaled rigid registration was used, the average mean reconstruction error of the leave-all-in study was changed to $1.03 \mathrm{~mm}$ (range: $0.9 \mathrm{~mm}$ to $1.3 \mathrm{~mm}$ ) and the average mean reconstruction error of the leave-four-out study was changed to $1.17 \mathrm{~mm}$ (range: $1.0 \mathrm{~mm}$ to $1.4 \mathrm{~mm})$.

Figure 11 shows an example of estimating the surface model reconstruction accuracy, where the ground truth model with a color-coded error distribution (middle column) is displayed together with the reconstructed surface model (right column) after a surface-based anisotropically scaled rigid registration was used to recover the unknown scale factor.
Table 2 Errors of reconstructing the surface models of the 3 lumbar vertebrae with complete structures

Vertebra Cadaver_1_L1 $\quad$ Cadaver_1_L2 $\quad$ Cadaver_1_L3

Leave-all-in study, when the anisotropically scaled rigid registration was used to recover the scale

$\begin{array}{llll}\text { Errors }(\mathrm{mm}) & 0.9 \pm 0.7 & 0.7 \pm 0.7 & 0.7 \pm 0.6\end{array}$

Leave-one-out study, when the anisotropically scaled rigid registration was used to recover the scale $\begin{array}{llll}\text { Errors }(\mathrm{mm}) & 0.9 \pm 0.8 & 0.8 \pm 0.8 & 0.8 \pm 0.7\end{array}$

Leave-all-in study, when the isotropically scaled rigid registration was used to recover the scale

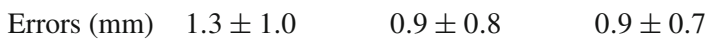

Leave-one-out study, when the isotropically scaled rigid registration was used to recover the scale Errors (mm) $1.4 \pm 1.1 \quad 1.0 \pm 0.9 \quad 1.1 \pm 0.8$

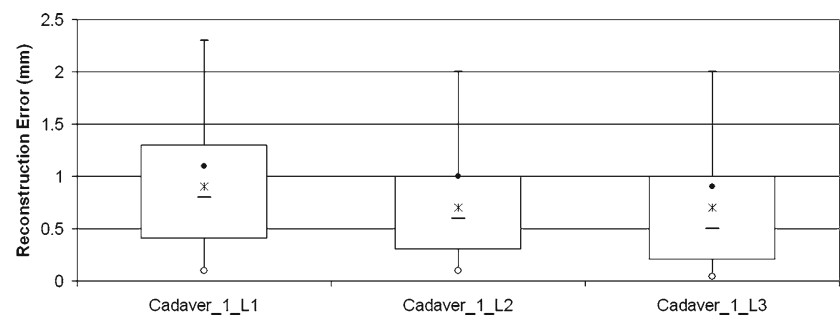

- $5 \%$ Percentile Error $-95 \%$ Percentile Error $*$ Mean Error - Median Error $\cdot$ RMS Error

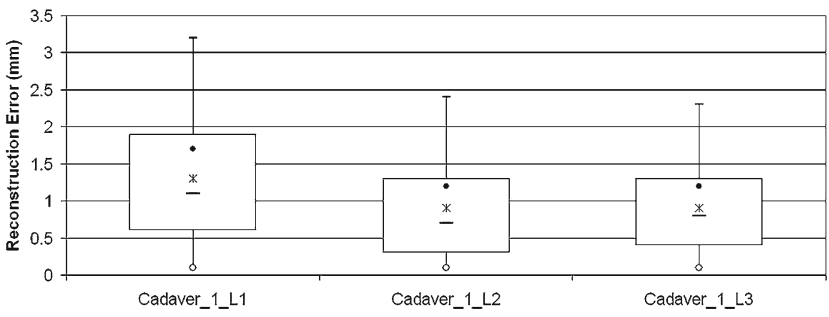

$\circ 5 \%$ Percentile Error $-95 \%$ Percentile Error $*$ Mean Error - Median Error - RMS Error

Fig. 10 Errors of reconstructing surface models of all 3 lumbar vertebrae with complete structures when different surface-based matching techniques were used to recover the unknown scale factors between the reconstructed surface models and the associated ground truths. Top row when a surface-based anisotropically scaled rigid registration was
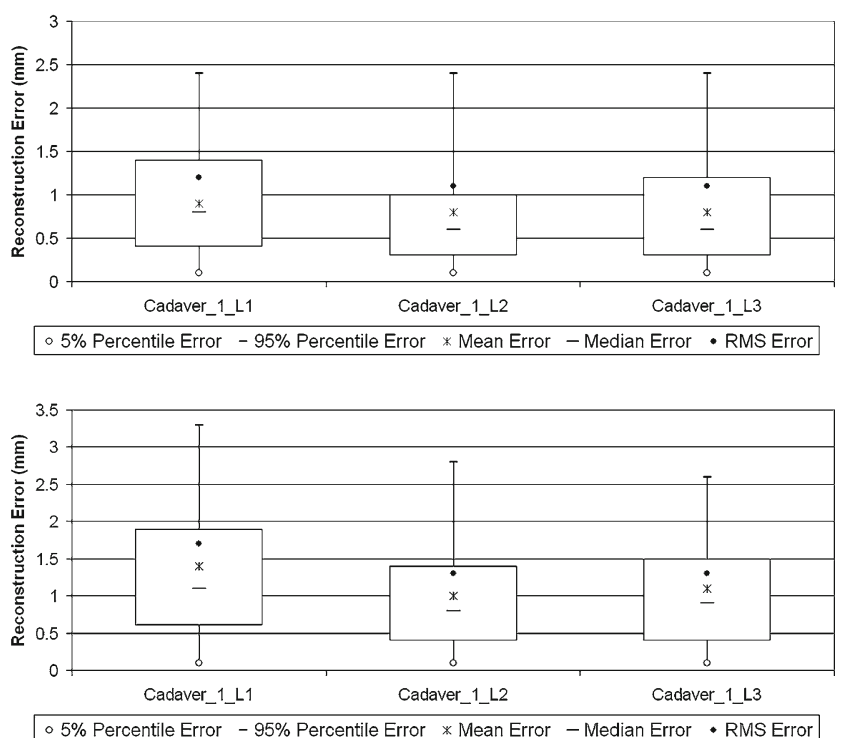

used; and bottom row when a surface-based isotropically scaled rigid registration was used. In both rows, the left column shows results of the leave-all-in study, while the right column shows the results of the leave-four-out study 
Fig. 11 Color-coded reconstruction error distribution. Left column: error bar; middle column: the ground truth model with the color-coded error distributions; right column the reconstructed model after a surface-based iterative affine registration was used to recover the unknown scale factor
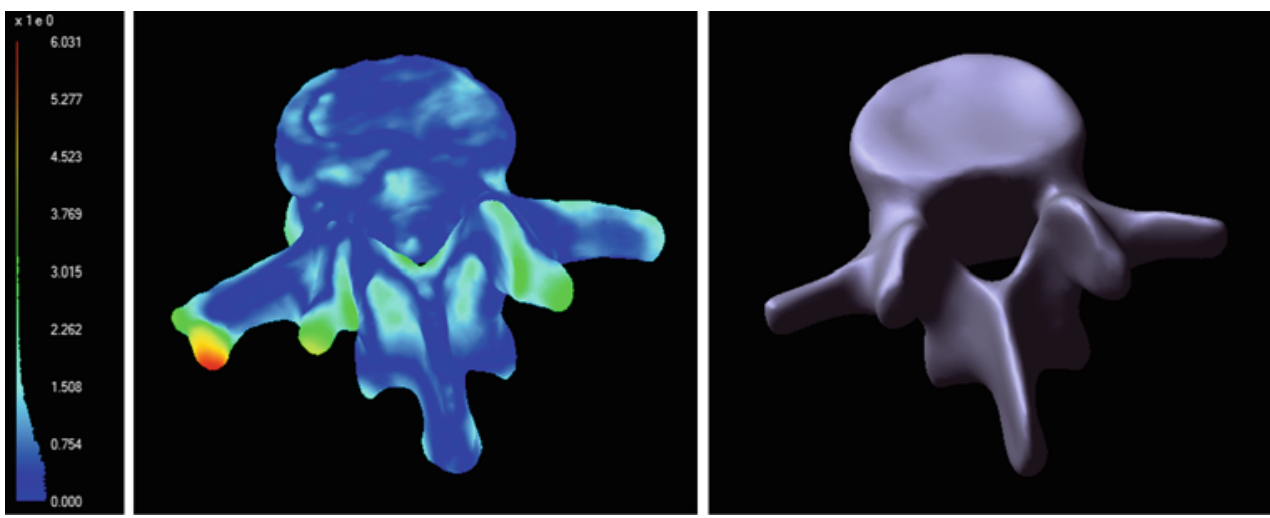

\section{Discussions and conclusions}

In this paper, we presented a single image-based 2D/3D reconstruction technique and showed its application to reconstruct a scaled, patient-specific 3D surface model of the lumbar vertebra from a single lateral fluoroscopic image. This single image-based $2 \mathrm{D} / 3 \mathrm{D}$ reconstruction technique is based on a hybrid 2D/3D deformable registration process combining a landmark-to-ray registration with a SSM-based $2 \mathrm{D} / 3 \mathrm{D}$ reconstruction. After evaluating the correspondenceestablishing quality of the two PDMs used in the present work, we validated the present $2 \mathrm{D} / 3 \mathrm{D}$ reconstruction technique by designing and conducting two additional experiments. The first one focused on evaluating the robustness and the accuracy of the present technique in reconstructing scaled surface models of 9 lumbar vertebrae with broken processes, while the second one aimed at evaluating the robustness and the accuracy of the present technique in reconstructing scaled surface models of 3 lumbar vertebrae with complete structures. In each experiment, we designed and conducted two studies, and in both studies, the present technique could successfully reconstruct scaled surface models of all test lumbar vertebrae. To evaluate the overall reconstruction accuracy, we investigated two different surface-based registration techniques to recover the unknown scale factors between the reconstructed surface models and their associated ground truths: the surface-based anisotropically scaled rigid registration and the surface-based isotropically scaled rigid registration. Our experimental results demonstrated that the present technique can reconstruct scaled surface models of all 12 test lumbar vertebrae in a reasonably good accuracy, i.e., the mean reconstruction errors were found to be in the range of $0.7-1.6 \mathrm{~mm}$. The overall reconstruction accuracy was slightly different when different surface-based registration techniques were used to estimate the unknown scale factors. It was also reasonable to observe that in both experiments, the results of the leave-all-in study were better than the other study. Such an observation indicated that the more shape variations that we integrated, the more accurate the present technique was. Another interesting finding was that the average accuracy of the experiment on reconstructing 3 lumbar vertebrae with complete structures was better than that of the experiment on reconstructing 9 lumbar vertebrae with broken transverse processes. One possible explanation for this finding is the differences in the number of training models used to construct the statistical shape models that were then used in the two different experiments for 2D/3D reconstructions.

It is worth noting that there are differences between the present technique and the single image-based 2D/3D reconstruction technique that we introduced previously [22]. Although both techniques can reconstruct a scaled, patientspecific surface model of a target structure from a single $\mathrm{X}$-ray image and both of them depend on the image-to-model correspondence-establishing algorithm that we introduced previously [28], they are different from each other on how the image contours are extracted and on how the statistical shape model is constructed. The image contours in the present technique are extracted by a semiautomatic segmentation tool based on the Livewire algorithm. Such a semiautomatic segmentation tool effectively exploits the synergy between a human operator who is superior in object recognition and the Livewire algorithm that is better in exact object delineation. Thus, it takes fewer labors and requires less user attention than the fully manual segmentation tool used in our previous works [20-22]. The local cost function that we designed for the Livewire algorithm, which is based on the multiscale Canny edge detector [27], can seamlessly handle both strong and weak edges in the fluoroscopic image due to its multiscale characteristic. Furthermore, unlike in our previous work [22], where the statistical shape model was constructed from a set of training surface models of one anatomical structure (the pelvis), here the statistical shape models were constructed from a set of training surface models of lumbar vertebrae across all five levels. The results from our experiment on evaluating the correspondence-establishing quality demonstrated the efficacy of the across-level lumbar vertebral SSMs used in the present study. 
The differences between the present technique and other works on reconstructing a patient-specific surface model of the vertebra should be discussed. Most of existing works $[6-11,14]$, except those introduced by Benameur et al. [12, 13], focused on the reconstruction of a surface model of the complete spine from two or more $\mathrm{X}$-ray radiographs, while in the present work, we were only interested in reconstructing a surface model of the lumbar vertebra due to our targeted application, i.e., the spine kinematics analysis. The main difference between the present technique and the methods introduced by Benameur et al. [12,13] lies in the optimization techniques that were used to reconstruct a patient-specific surface model. More specifically, in Benameur et al. [12,13], a PDM of scoliotic vertebrae was fitted to two calibrated $\mathrm{X}$-ray radiographs by simultaneously optimizing both the shape and the pose parameters, while in the present work, we sequentially optimized the shape and the pose parameters. Furthermore, in the present work, the surface model obtained after the statistical instantiation stage was further refined by the regularized shape deformation algorithm. The advantages of integrating this additional stage into the present technique over other existing attempts to instantiate a patientspecific surface model from a statistical shape model were explained in detail in our previous work [28]. Briefly speaking, such integration enables the present technique to handle more complicated shape variation of any future instance [28].

While accurate, the present approach has limitations related with the number of training models used to construct the statistical shape models and the number of validation cases. The accuracy of the present approach depends not only upon how accurate the image-to-model correspondences can be established but also upon how well the unknown, patient-specific shape variation can be covered by the statistical shape model that is constructed from a fixed number of training models. Although the image-to-model correspondence-establishing process has been thoroughly validated in our previous works [20-22,28] as well as in the present work, both statistical shape models used in the present study were constructed from a limited number of training lumbar vertebral models (39 for the complete-vertebra PDM and 11 for the broken-vertebra PDM). Furthermore, the validation of the present approach, though successful, was only conducted on datasets of 12 lumbar vertebrae. Thus, the results reported in this paper are regarded still preliminary, and more thorough validation study is needed before it can be transferred to a routine usage. Nonetheless, the experiment results from the present study demonstrate the efficacy of the present approach, and the prediction power of the present approach can be enhanced in the future by incorporating more training models into the statistical shape model and/or by constructing a patient-oriented statistical shape model.

In summary, this paper presented a statistical shape model-based technique to reconstruct a scaled, patient- specific surface model of a lumbar vertebra from a single lateral fluoroscopic image. Future work will focus on applying the reconstructed model for 3D kinematic analysis of lumbar vertebrae. The developed method also holds potentials in surgical planning and navigation applications.

Acknowledgments The authors gratefully acknowledge the financial support from the Swiss National Science Foundation through the National Centers of Competence in Research CO-ME (G. Zheng and L.-P. Nolte) and National Research Program NRP 53 (S. J. Ferguson). The authors would also like to thank the anonymous reviewers for their constructive comments and suggestions. The authors thank P. Thistlethwaite and P. Gédet for the fluoroscopic imaging.

\section{References}

1. Muggleton JM, Allen R (1997) Automatic location of vertebrae in digitized videofluoroscopic images of the lumbar spine. Med Eng Phys 19:77-89

2. Zheng Y, Nixon MS, Allen R (2003) Lumbar spine visualization based on kinematic analysis from videofluoroscopic imaging. Med Eng Phys 25:171-179

3. Wang S, Passias P, Li G, Li G, Wood K (2009) Measurement of vertebral kinematics using noninvasive image matching methodvalidation and application. Spine 33:E355-E361

4. Bifulco P, Sansone M, Cesarelli M, Allen R, Bracale M (2002) Estimation of out-of-plane vertebra rotations on radiographic projections using ct data: a simulation study. Med Eng Phys 24:295-300

5. Hanson GR, Suggs JS, Freiberg AA, Durbhakula S, Li G (2006) Investigation of in vivo 6DOF total knee arthroplasty kinematics using a dual orthogonal fluoroscopic system. J Orthop Res 24:974-981

6. Aubin CE, Descrimes JL, Dansereau J, Skalli W, Lavaste F, Labelle H (1995) Geometrical modeling of the spine and thorax for biomechanical analysis of scoliotic deformities using finite element method. Ann Chir 49:749-761

7. Mitton D, Landry C, Vron S, Skalli W, Lavaste F, De Guise JA (2000) 3D Reconstruction method from biplanar radiography using non-stereocorresponding points and elastic deformable meshes. Med Bio Eng Comput 38:133-139

8. Pomero V, Mitton D, Laporte S, De Guise JA, Skalli W (2004) Fast accurate stereoradiographic 3D-reconstruction of the spine using a combined geometric and statistic model. Clin Biomech 19:240247

9. Humbert L, De Guise JA, Aubert B, Godbout B, Skalli W (2009) 3D Reconstruction of the spine from biplanar X-rays using parametric models based on transversal and longitudinal inferences. Med Eng Phys 31:681-687

10. Dumas R, Blanchard B, Carlier R, de Loubresse CG, Le Huec J-C, Marty C, Moinard M, Vital J-M (2009) A semi-automated method using interpolation and optimization for the $3 \mathrm{D}$ reconstruction of the spine from bi-planar radiography: a precision and accuracy study. Med Bio Eng Comput 46:85-92

11. Kadoury S, Cheriet F, Labelle H (2009) Personalized X-ray 3D reconstruction of the scoliotic spine from hybrid statistical and image-models. IEEE Trans Med Imaging 28:1422-1435

12. Benameur S, Mignotte M, Parent $S$ et al (2003) $3 d / 2 d$ registration and segmentation of scoliotic vertebra using statistical models. Comput Med Imag Grap 27:321-337

13. Benameur S, Mignotte M, Labelle H, De Guise JA (2005) A hierarchical statistical modeling approach for the unsupervised 3-D 
biplanar reconstruction of the scoliotic spine. IEEE Trans Biomed Eng 12:2041-2057

14. Boisvert J, Cheriet F, Pennec X, Labelle H, Ayache N (2008) Articulated spine models for 3-D reconstruction from partial radiographic data. IEEE Trans Biomed Eng 55:2565-2574

15. Kendall D (1989) A survey of the statistical theory of shape. Stat Sci 4:87-120

16. Small CG (1996) The statistical theory of shape. Springer series in statistics. Springer, New York

17. Dryden I, Mardia K (1998) Statistical shape analysis. Wiley series in probability and statistics. Wiley, New York

18. Turk M, Pentland A (1991) Eigenfaces for recognition. J Cogn Neurosci 3:71-86

19. Cootes TF, Hill A, Taylor CJ, Haslam J (1994) The use of active shape models for locating structures in medical images. Image Vis Comput 12:355-366

20. Zheng G, Gonzalez Ballester MA, Styner M, Nolte L-P (2006) Reconstruction of patient-specific $3 \mathrm{~d}$ bone surface from $2 \mathrm{~d}$ calibrated fluoroscopic images and point distribution model. In: MICCAI'06 vol. Part I, LNCS 4190, pp 25-32

21. Zheng G, Schumann S (2009) 3D reconstruction of a patient-specific surface model of the proximal femur from calibrated X-ray radiographs: a validation study. Med Phys 36:1155-1166

22. Zheng G (2009) Statistically deformable $2 d / 3 d$ registration for accurate determination of post-operative cup orientation from single standard X-ray radiograph. In: MICCAI'09 vol. Part I, LNCS 5761, pp 820-827

23. Barrett WA, Mortensen EN (1997) Interactive live-wire boundary extraction. Med Image Anal 1:331-341

24. Toussaint N, Souplet J-C, Fillard P (2007) Image matching as a diffusion process: an analogy with maxwell's demons. In:
MICCAI'07 Workshop on interaction in medical image and visualization

25. Jolliffe IT (2002) Principal component analysis, 2nd edn. Springer series in statistics. Springer, New York

26. Hofstetter R, Slomczykowski M, Sati M, Nolte L-P (1999) Fluroroscopy as an imaging means for computer-assisted surgical navigation. Comput Aided Surg 4:65-76

27. Canny J (1986) A computational approach to edge detection. IEEE Trans Pattern Anal Mach Intell 8:679-714

28. Zheng G, Gollmer S, Schumann S, Dong X, Feilkas T, Gonzalez Ballester MA (2009) A 2D/3D correspondence building method for reconstruction of a patient-specific $3 \mathrm{D}$ bone surface model using point distribution models and calibrated X-ray images. Med Image Anal 13(6):883-899

29. Hertzmann A, Zorin D (2000) Illustrating smooth surfaces. In: Proceedings of the 27th annual conference on computer graphics and interactive techniques (SIGGRAPH'00). ACM Press/AddisonWesley Publishing Cp., New York, pp 517-526

30. Besl P, McKay ND (1992) A method for registration of $3 d$ shapes. IEEE Trans Pattern Anal Mach Intell 14:239-256

31. Aspert N, Santa-Cruz D, Ebrahimi T (August 2002) MESH: Measuring errors between surfaces using the Hausdorff distance. In: Proceedings of 2002 IEEE conference on Multimedia and Expo (ICME 2002), Lausanne, vol 1, pp 705-708

32. Davies RH (2002) Learning shape: optimal models for analyzing natural variability. Doctoral Dissertation, University of Manchester

33. Styner MA, Rajamnai KT, Nolte L-P, Zsemlye G, Székely G, Taylor CJ, Davies RH (2003) Evaluation of 3D correspondences methods for model building. In: Proceeding of IPMI 2003, LNCS 2732, pp 63-75 